\title{
Evaluation of Micro-focused Ultrasound for Lifting and Tightening the Face
}

\author{
In Ho Lee, Seung Min Nam, \\ Eun Soo Park, Yong Bae Kim \\ Department of Plastic and Reconstructive \\ Surgery, College of Medicine, \\ Soonchunhyang University, Bucheon, \\ Korea
}

This work was supported by the Soonchunhyang University Research Fund.

No potential conflict of interest relevant to this article was reported.
Background Micro-focused ultrasound (MFU) has developed as an effective, noninvasive, skin-tightening method. However, certain factors have limited its replacement of invasive surgical procedures, including a relative lack of efficacy, persistence, and reliability. The purpose of this study was to evaluate the efficacy and safety of MFU for noninvasive skin tightening and to determine how long the skin tightening can be maintained.

Methods Between October 2013 and November 2014, 41 patients with sagging and laxity of the facial skin were treated with MFU. The treatment was performed following the manufacturer's recommended protocol that called for 300 treatment lines. We evaluated the patients using an automatic skin diagnosis system at pretreatment, and 2 and 4 months after treatment.

Results Of the 41 patients treated using MFU, 3 patients were lost to follow-up for nonstudy-related reasons. In our study, 38 patients (1 male and 37 female) were evaluated and ranged in age from 37 to 52 years. The median skin grade scores were 5 at pretreatment, 3 at 2 months posttreatment and 3 at 4 months posttreatment. After comparing pretreatment and 2 months posttreatment, pretreatment and 4 months posttreatment, and both 2 and 4 months posttreatment, there were statistically significant differences $(\mathrm{P}<0.01)$.

Conclusions This study suggests that the aging face, with wrinkling and sagging, can be improved using MFU, while minimizing injury to the epidermis and dermis.

Keywords Micro-focused Ultrasound, Aging face, Lifting

\section{INTRODUCTION}

The signs of aged facial skin are not only fine lines and surface irregularities, but also sagging and wrinkling [1]. Noninvasive skin tightening is superior to invasive or surgical skin tightening in terms of rapid return to work, short recovery time, and low risk of adverse events. Because of these advantages, patients who desire a

Received: May 13, 2015 Revised: May 27, 2015 Accepted: May 27, 2015 Correspondence: Seung Min Nam Department of Plastic and Reconstructive, Soonchunhyang University Bucheon Hospital, 170 Jomaruro, Wonmi-gu, Bucheon 420-767, Korea. E-mail: zodiac1003@naver.com

Copyright @ 2015 The Korean Society for Aesthetic Plastic Surgery.

This is an Open Access article distributed under the terms of the Creative Commons Attribution Non-Commercial License (http://creativecommons.org/licenses/by-nc/3.0/) which permits unrestricted non-commercial use, distribution, and reproduction in any medium, provided the original work is properly cited. www.e-aaps.org skin-tightening procedure prefer noninvasive skin tightening to invasive or surgical skin tightening $[1,2]$.

To meet the demand of patients for noninvasive skin tightening, numerous devices have been developed. Laser and radiofrequency devices have been developed to resolve skin wrinkling and sagging [1-8]. Recently, micro-focused ultrasound (MFU) was developed as an effective noninvasive skin-tightening method. MFU is able to heat tissue greater than $60^{\circ} \mathrm{C}$ and produce a small thermal coagulation zone $\left(<1 \mathrm{~mm}^{3}\right)$ to reach the mid- to deep reticular layers of the dermis and subdermis while minimizing overlying papillary dermal and epidermal injury [9-11]. The delivery of MFU to a targeted zone in the superficial musculoaponerotic system (SMAS) provokes immediate contracture of denatured collagen and the initiation of neocollagenesis and collagen remodeling [10,12]. This action of MFU provokes noninvasive skin tightening and lifting of sagging facial skin. 
Certain factors have limited its replacement of invasive surgical procedures, including a relative lack of efficacy, persistence, and reliability. The purpose of this study was to evaluate the efficacy and safety of MFU for noninvasive skin tightening and to determine how long the skin tightening can be maintained.

\section{METHODS}

Between October 2013 and November 2014, 41 patients with sagging and laxity of the facial skin were treated with MFU using 4$\mathrm{MHz}, 4.5 \mathrm{~mm}$ and 7-MHz, $3.0 \mathrm{~mm}$ depth transducers (Ultraformer $^{\circledR}$, Classys Inc., Seoul, Korea). Treatment was performed following the manufacturer's recommended protocol that called for 300 treatment lines. Patients with active systemic or local infections, local skin diseases that might alter wound healing, history of psychiatric illness, and soft tissue augmentation material were excluded from this study.

\section{Pretreatment preparation}

Five percent lidocaine, as a topical anesthetic ointment (EMLA, AstraZeneca, Sdertlje, Sweden), was applied to the face for $45 \mathrm{~min}$ utes before the procedure. The ointment was washed off with mild soap and water immediately before the procedure.

\section{Ultrasound exposure protocol}

The ultrasound gel was applied to the skin. The transducer was placed firmly on the targeted skin surface and pressed uniformly for compling to the skin. Treatment exposure was initiated (4-MHz, $4.5 \mathrm{~mm}$ depth transducers; $0.9 \mathrm{~J} / \mathrm{mm}^{2}$ and 7-MHz, $3.0 \mathrm{~mm}$ depth transducers; $0.8 \mathrm{~J} / \mathrm{mm}^{2}$ ), with a line of individual ultrasound pulses being delivered within approximately 2 seconds. Then, the transducer slid to the next location and was repositioned 3 to $5 \mathrm{~mm}$ laterally such that it was adjacent and parallel to the previous treatment line. Complete treatment of the face required 15 to 20 minutes.

\section{Posttreatment care}

The ultrasound gel was washed off. Patients experienced mild redness and swelling that could persist for several days. Patients were instructed to visit our hospital promptly if they encountered any other adverse effects.

\section{Outcome evaluation}

We evaluated the patients using an automatic skin diagnosis system (A-One Lite ${ }^{\circledR}$, BOMTECH Electronics Co., Seoul, Korea) at

Table 1. Patients Characteristics

\begin{tabular}{lc}
\hline Characteristic & Value \\
\hline Sex (Female, Male) & 37,1 \\
Mean Age (range) & $46(37-52)$ \\
\hline
\end{tabular}

pretreatment, and 2 and 4 months after treatment. The automatic skin diagnosis system evaluated skin laxity using a scanner. The sagging and laxity of the skin were graded from 1 to 6 using the system. A high skin grade score means that the sagging and laxity of the skin are severe. The clinician examined the skin for evidence of edema, erythema, hypopigmentation, and hyperpigmentation after treatment.

\section{Statistical analysis}

Statistical analyses were performed using SPSS version 20.0 (SPSS Inc., Chicago, IL, USA). The Friedman test was used to compare the grade scores of patients at pretreatment, and 2 and 4 months after treatment. A P value less than 0.05 was considered statistically significant.

\section{RESULTS}

All patients were treated using MFU and three patients were lost to follow-up for non-study related reasons. In our study, 38 patients (1 male and 37 female) were evaluated and ranged in age from 37 to 52 years (Table 1 ). Thirty-five patients immediately presented with slight erythema and edema after treatment, and three patients immediately presented with moderate erythema and edema after treatment. In all affected patients, both erythema and edema completely resolved by 2 days after treatment. Two patients presented

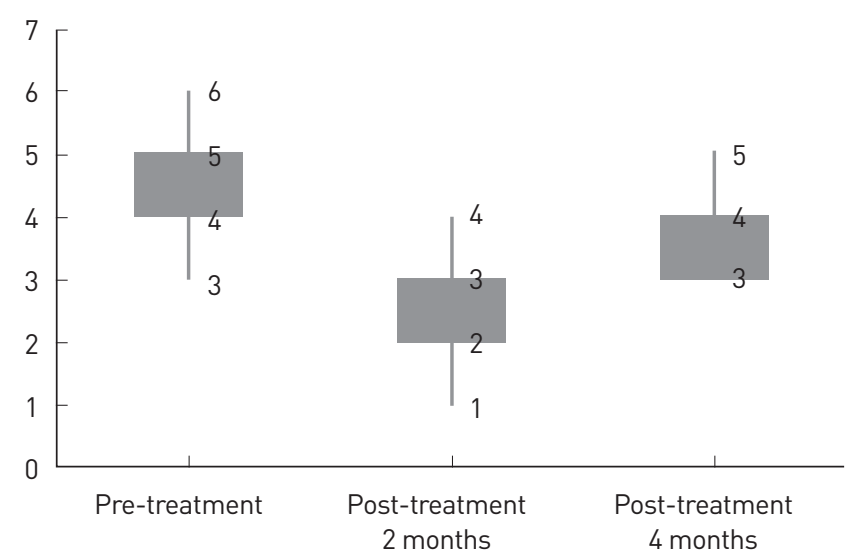

Fig. 1. Comparisons of skin grade scores at pretreatment and 2 months posttreatment, pretreatment and 4 months posttreatment 4 months, and 2 and 4 months posttreatment.

Table 2. The skin grade score

\begin{tabular}{lcccc}
\hline Time & $\begin{array}{c}\text { Pre- } \\
\text { treatment } \\
\text { (Median) }\end{array}$ & $\begin{array}{c}\text { Post- } \\
\text { treatment } \\
\text { 2 months } \\
\text { (Median) }\end{array}$ & $\begin{array}{c}\text { Post- } \\
\text { treatment } \\
\text { 4 months } \\
\text { (Median) }\end{array}$ & P-value ${ }^{*,+, \neq}$ \\
\hline Skin grade score & $5^{*, \ddagger}(4-5)$ & $3^{*,+}(2-3)$ & $3^{+, \neq}(3-4)$ & $<0.01$ \\
\hline
\end{tabular}

*,+, P-value by Wilcoxon signed rank test. 
with red linear striations of the check after treatment with the $3 \mathrm{~mm}$ transducer. They were treated using focal cooling without sequelae such as pigmentation and textural abnormalities. Hypopigmentation, hyperpigmentation, ulceration, and erosion were not present in any patients. There were no adverse events, such as nerve or muscle dysfunction, severe pain, bruising, and bleeding.
The median skin grade scores were $5(4-5)$ at pretreatment, 3 (23 ) at 2 months posttreatment, and 3 (3-4) at 4 months posttreatment (Fig. 1 and Table 2). After comparing pretreatment and 2 months posttreatment, pretreatment and 4 months posttreatment, and both 2 and 4 months posttreatment, there was a statistically significant difference in skin grade score $(\mathrm{P}<0.01)$ (Fig. 2 and 3$)$.
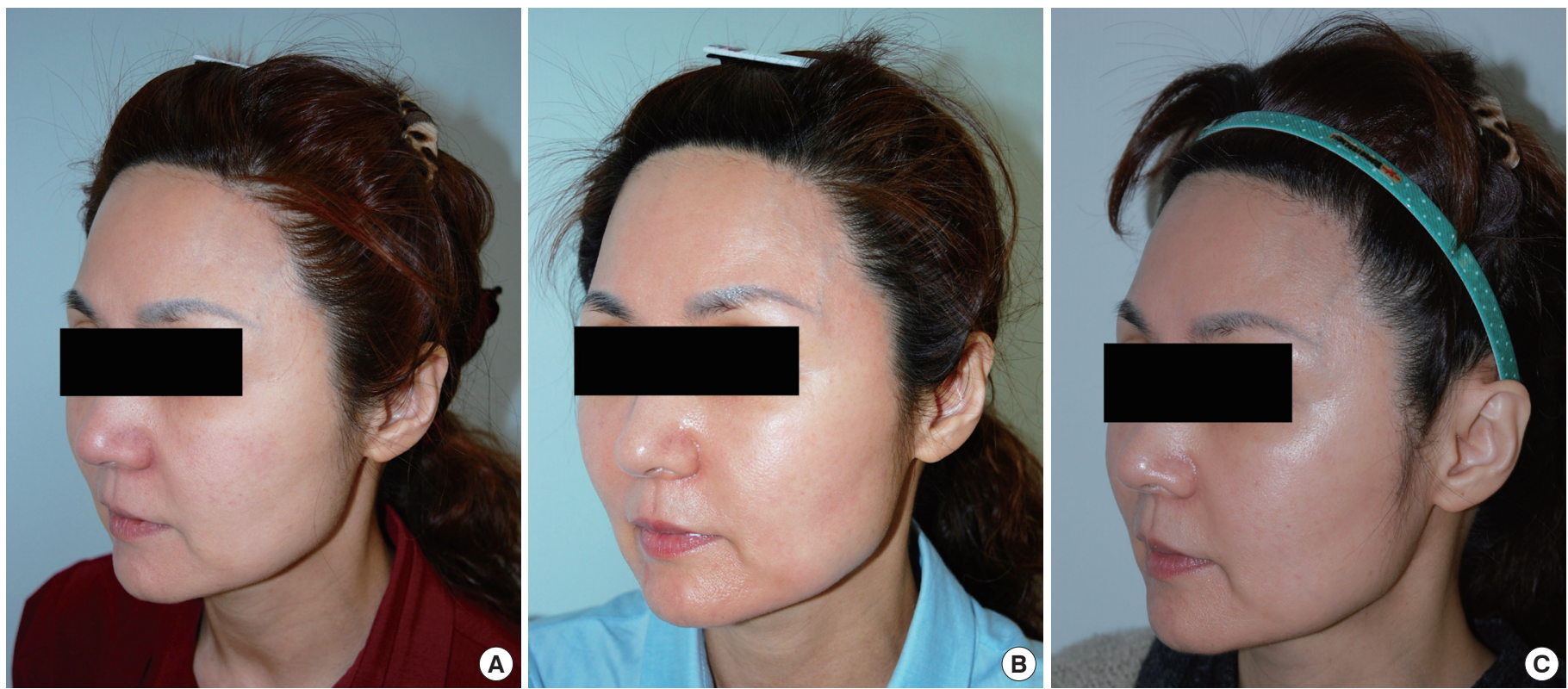

Fig. 2. A 46-year-old female patient with moderate skin sagging and wrinkling. At pretreatment, she was examined by the automatic skin diagnosis system and was given a skin grade score of 5 (A). At 2 months posttreatment, the skin grade score was 2 (B). At 4 months posttreatment, the skin grade score was 4 (C).
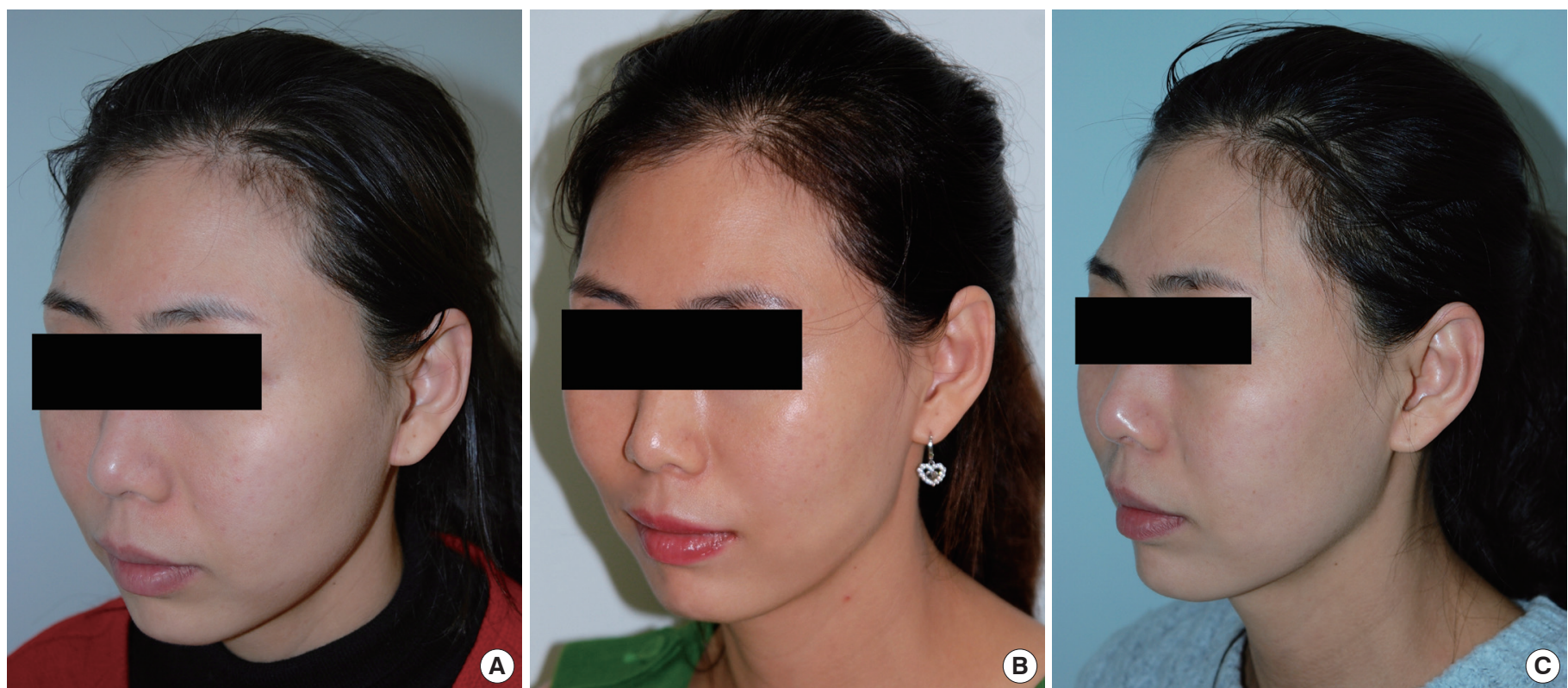

Fig. 3. A 38-year-old female patient with moderate skin sagging and wrinkling. At pretreatment, she was examined by the automatic skin diagnosis system and was given a skin grade score of 4 (A). At 2 months posttreatment, the skin grade score was 2 (B). At 4 months posttreatment, the skin grade score was 3 (C). 


\section{DISCUSSION}

The SMAS consists of viscous, elastic fibers and extracellular matrix $[10,13,14]$. It is associated with specific facial muscles, such as the platysma, orbicularis oculi, and levator labii superioris. Collagen within SMAS decreases 6\% every decade [10]. This decrease in collagen contributes to a prominent nasolabial fold, and hooding of the brow and jowl $[10,15,16]$.

To minimize posttreatment adverse events, clinicians have developed various nonablative skin-tightening procedures to induce collagen shrinkage and remodeling $[3,6,17]$. Furthermore, ultrasound is able to penetrate into the subdermis layer and SMAS, and induce thermal coagulation to avoid undesired posttreatment adverse events compared with carbon-dioxide laser resurfacing [1719].

Ultrasound energy has characteristics that are suitable for skin lifting and tightening. First, it is believed that ultrasound energy can be transmitted into the deeper subcutaneous layer of the face or even the SMAS, and is the most effective method for skin lifting and tightening [13,14,20-23]. Second, both the epidermis and dermis can be protected from ultrasound energy during its transmission, reducing the risk of advertent cutaneous layers [1].

Ultrasound used in medicine is classified into two types. One is high-intensity focused ultrasound (HIFU) and the other is MFU. HIFU uses high energy and is mainly used for nonsurgical ablation of tumors. HIFU can also be used to ablate adipose tissue for body contouring [10]. MFU uses much lower energy to treat the superficial layer of the skin [9] and is able to elevate the local temperature higher than $60^{\circ} \mathrm{C}$ to cause collagen contracture [24]. When energy is targeted to discrete areas within dermal and subdermal tissues, MFU induces discrete thermal coagulation zones while sparing adjacent nontarget tissues $[9,11,12,25]$. In addition, the heat induces the denaturation and contraction of collagen fibers in the subcutaneous fat layer [26].

According to the results of our study, skin tightening at 2 and 4 months posttreatment was improved compared to pretreatment. However, skin tightening at 2 months posttreatment was better than at 4 months posttreatment, suggesting the efficacy of MFU gradually decreases treatment. Based on our results, we recommend that retreatment should be performed after 3 months for greater efficacy.

Our study had limitations. First, our study did not include patients who had severe skin sagging and wrinkling. We recommended the surgical face-lift procedure for these patients. Second, the posttreatment results were evaluated with an automatic skin diagnosis system, but the reliability of the system has not been established. Therefore, discrepancies may occur between the automatic skin diagnosis system and realistic skin conditions. Third, our study did not include any histologic evaluations. Fourth, the MFU device that we used in our study is not capable of clearly imaging the tar- geted facial anatomy. We cannot ensure proper acoustic coupling between the transducer and skin before the application of MFU energy. Despite these limitations, the results were evaluated objectively.

\section{CONCLUSION}

This study suggests that the aging face, with wrinkling and sagging, can be improved using MFU, while minimizing injury to the epidermis and dermis. In addition, retreatment is recommended after 3 months to maintain the efficacy of the results.

\section{PATIENT CONSENT}

Patients provided written consent for the use of their images.

\section{REFERENCES}

1. Alam M, White LE, Martin N, et al. Ultrasound tightening of facial and neck skin: a rater-blinded prospective cohort study. J Am Acad Dermatol 2010;62:262-9.

2. Dover JS, Zelickson B. Results of a survey of 5,700 patient monopolar radiofrequency facial skin tightening treatments: assessment of a lowenergy multiple-pass technique leading to a clinical end point algorithm. Dermatol Surg 2007;33:900-7.

3. Fitzpatrick R, Geronemus R, Goldberg D, et al. Multicenter study of noninvasive radiofrequency for periorbital tissue tightening. Lasers Surg Med 2003;33:232-42.

4. Fitzpatrick RE, Goldman MP, Satur NM, et al. Pulsed carbon dioxide laser resurfacing of photo-aged facial skin. Arch Dermatol 1996;132: 395-402.

5. Goco PE, Stucker FJ. Subdermal carbon dioxide laser cutaneous contraction. Arch Facial Plast Surg 2002;4:37-40.

6. Kim KH, Geronemus RG. Nonablative laser and light therapies for skin rejuvenation. Arch Facial Plast Surg 2004;6:398-409.

7. Kirsch KM, Zelickson BD, Zachary CB, et al. Ultrastructure of collagen thermally denatured by microsecond domain pulsed carbon dioxide laser. Arch Dermatol 1998;134:1255-9.

8. Zelickson BD, Kist D, Bernstein E, et al. Histological and ultrastructural evaluation of the effects of a radiofrequency-based nonablative dermal remodeling device: a pilot study. Arch Dermatol 2004;140:204-9.

9. Fabi SG. Noninvasive skin tightening: focus on new ultrasound techniques. Clin Cosmet Investig Dermatol 2015;8:47-52.

10. Fabi SG, Goldman MP. Retrospective evaluation of micro-focused ultrasound for lifting and tightening the face and neck. Dermatol Surg 2014;40:569-75.

11. Laubach HJ, Makin IR, Barthe PG, et al. Intense focused ultrasound: evaluation of a new treatment modality for precise microcoagulation within the skin. Dermatol Surg 2008;34:727-34.

12. White WM, Makin IR, Barthe PG, et al. Selective creation of thermal 
injury zones in the superficial musculoaponeurotic system using intense ultrasound therapy: a new target for noninvasive facial rejuvenation. Arch Facial Plast Surg 2007;9:22-9.

13. Ghassemi A, Prescher A, Riediger D, et al. Anatomy of the SMAS revisited. Aesthetic Plast Surg 2003;27:258-64.

14. Har-Shai Y, Bodner SR, Egozy-Golan D, et al. Mechanical properties and microstructure of the superficial musculoaponeurotic system. Plast Reconstr Surg 1996;98:59-70.

15. Kikkawa DO, Lemke BN, Dortzbach RK. Relations of the superficial musculoaponeurotic system to the orbit and characterization of the orbitomalar ligament. Ophthal Plast Reconstr Surg 1996;12:77-88.

16. Psillakis JM, Rumley TO, Camargos A. Subperiosteal approach as an improved concept for correction of the aging face. Plast Reconstr Surg 1988;82:383-94.

17. Fabi SG, Massaki A, Eimpunth S, et al. Evaluation of microfocused ultrasound with visualization for lifting, tightening, and wrinkle reduction of the decolletage. J Am Acad Dermatol 2013;69:965-71.

18. Alster TS, Tanzi E. Improvement of neck and cheek laxity with a nonablative radiofrequency device: a lifting experience. Dermatol Surg 2004; 30:503-7.

19. Kennedy JE, Ter Haar GR, Cranston D. High intensity focused ultrasound: surgery of the future? Br J Radiol 2003;76:590-9.
20. Har-Shai Y, Sela E, Rubinstien I, et al. Computerized morphometric quantitation of elastin and collagen in SMAS and facial skin and the possible role of fat cells in SMAS viscoelastic properties. Plast Reconstr Surg 1998;102:2466-70.

21. Mendelson BC. Surgery of the superficial musculoaponeurotic system: principles of release, vectors, and fixation. Plast Reconstr Surg 2001; 107:1545-52.

22. Mitz V, Peyronie M. The superficial musculo-aponeurotic system (SMAS) in the parotid and cheek area. Plast Reconstr Surg 1976;58:80-8.

23. Thaller SR, Kim S, Patterson H, et al. The submuscular aponeurotic system (SMAS): a histologic and comparative anatomy evaluation. Plast Reconstr Surg 1990;86:690-6.

24. Hayashi K, Thabit G, 3rd, Massa KL, et al. The effect of thermal heating on the length and histologic properties of the glenohumeral joint capsule. Am J Sports Med 1997;25:107-12.

25. Gliklich RE, White WM, Slayton MH, et al. Clinical pilot study of intense ultrasound therapy to deep dermal facial skin and subcutaneous tissues. Arch Facial Plast Surg 2007;9:88-95.

26. Ferraro GA, De Francesco F, Nicoletti G, et al. Histologic effects of external ultrasound-assisted lipectomy on adipose tissue. Aesthetic Plast Surg 2008;32:111-5. 